\title{
A Case of Acute Generalised Exanthematous Pustulosis after Unprotected Sexual Intercourse on Holidays in Bali
}

\author{
Tristan Blake Salvatore Scuderi \\ Department of Dermatology, Royal Brisbane and Women's Hospital, \\ Brisbane, QId., Australia
}

\section{Key Words}

Acute generalised exanthematous pustulosis · Ibuprofen

\begin{abstract}
We present the case of a 43-year-old man with a clinical diagnosis of acute generalised exanthematous pustulosis in the setting of a recent 9-day holiday to Bali, Indonesia, to visit a female partner whom he had visited five times this year for unprotected sexual intercourse and from whom he had contracted a sexually transmitted infection. He also reports having taken some Indonesian cold and flu medications and ibuprofen.
\end{abstract}

\section{Introduction}

Acute generalised exanthematous pustulosis (AGEP) is a significant adverse cutaneous reaction, most often provoked by drugs and acute infections $[1,2]$. The clinical hallmark is the presence of multiple disseminated sterile non-follicular pustules on an erythematous background, predominantly in the folds and/or the face. It is associated with fever, neutrophilia and sometimes eosinophilia. The disease is characterised by an acute onset and typically a spontaneous resolution within 2 weeks [2]. 
Blake et al.: A Case of Acute Generalised Exanthematous Pustulosis after Unprotected Sexual Intercourse on Holidays in Bali

\section{Case Report}

A 43-year-old man presented to a rural hospital in Australia for lethargy, fever, arthralgia, chills, peri-orbital oedema and an erythematous pustular rash increasing in size over his hands, back, chest and face for approximately $48 \mathrm{~h}$. He was a fly-in fly-out worker in the catchment area of the hospital. He mentioned holidaying in Bali, Indonesia, for 9 days to visit a female partner with whom he had unprotected sexual intercourse. The symptoms started promptly upon his return. All in all, he had visited his partner five times this calendar year. He reported to have contracted genital herpes from this partner on his first visit and had sought treatment with antivirals (acyclovir) upon his return to Australia and had remained on them ever since. He also mentioned that both he and his partner had been monogamous since the day they met each other. Furthermore, he described some recent coryzal symptoms towards the end of his holiday, for which he bought some local cold and flu medication, the name and ingredients of which being unknown but being somewhat 'like Sudafed ${ }^{\circledR}$ '. He also took some Nurofen ${ }^{\circledR}$ tablets (ibuprofen) in Bali and upon returning to Australia. Apart from cystic acne 25 years ago and intermittently taking ibuprofen, the patient reported no significant past medical history.

At this rural hospital, he continued to decline systemically (fever $>39.5^{\circ} \mathrm{C}$, heart rate $>125 \mathrm{bpm}$ ) over $36 \mathrm{~h}$ and was flown to a tertiary referral hospital in Brisbane. On arrival, he had widespread pustules on his face, neck, back, chest, upper and lower limbs (fig. 1, fig. 2, fig. 3). It was noted that he was also erythematous and had an oedematous face. He described only a mild itch and denied any genitourinary symptoms. Some inguinal and cervical lymphadenopathy and minimal oral involvement were observed. He had a raised white blood cell count (13.2), neutrophilia (12.17), a mild lymphocytosis (0.56), a raised Creactive protein level (273), and was started immediately on intravenous piperacillin/tazobactam three times daily, intravenous vancomycin twice daily and intravenous acyclovir three times daily with a suspected but yet unknown infection.

He had negative serologies over the initial week for the following infections: streptococcus, syphilis, Ross River virus, Barmah Forest virus, alphavirus, Rickettsia rickettsii, measles, mumps, leptospirosis, parvovirus B19, enterovirus, varicella and HIV.

Histological examination of biopsies revealed conflicting features. A biopsy of the hand showed evidence of spongiotic dermatitis with eosinophilic dermal infiltrates and occasional intra-epidermal vesicles harbouring Langerhans cells. A second specimen of the abdomen revealed acute neutrophilic suppurative folliculitis. Direct immunofluorescence of the abdomen biopsy was negative. These results were considered inconsistent with the clinical impression of AGEP held at that time. Further biopsies were then taken from his chest and left thigh, which showed spongiform subcorneal pustules accompanied by dermal inflammatory infiltrates and perivascular infiltrates with occasional eosinophils reported as 'acute neutrophilic folliculitis'. Acanthosis and papillomatosis were not observed. A biopsy of the right foot favoured a spongiotic hypersensitivity reaction with a vasculitic component.

Clinically, his symptoms appeared to indicate AGEP at this stage, and given that serologies up until this point were negative, he was started on oral prednisone $0.5 \mathrm{mg} / \mathrm{kg}$ and topical betamethasone dipropionate $0.05 \%$ ointment twice daily, with a very good effect over the proceeding 5 days. It was only on day 6 of this treatment that, with the help of the infectious diseases team, a positive scrub typhus (Orientia tsutsugamushi) $\operatorname{Ig}_{\text {total }}$ serology was confirmed, with a titre of 1:128. This was not considered a primary infection, and paired tests 1 and 4 weeks later showed that the titre was stable and that this was likely an old rickettsial infection or a cross-reaction. This was also paired with scrub typhus and spotted fever PCR testing, which was also negative. Prednisone was weaned down to half the dose 
Blake et al.: A Case of Acute Generalised Exanthematous Pustulosis after Unprotected Sexual Intercourse on Holidays in Bali

over the following 5 days, and he was discharged home on day 13 of admission (significantly improved on emollients and weaning dose of prednisone). Post-pustular desquamation was observed around this time. An outpatient review 12 days following discharge revealed a slight flare-up again at home after taking ibuprofen tablets for $48 \mathrm{~h}$ for aches and pain. However, this resolved promptly with the cessation of the non-steroidal anti-inflammatory drug.

\section{Discussion}

This case represents a challenging diagnostic case with a variety of potential precipitants. Clinically, this patient had all the hallmark symptoms of AGEP as described in the literature [1-3] but was confused initially with having an acute infectious disease given his preceding history. A wide variety of other cutaneous diseases or reactions can cause pustular eruptions, and most of them can be easily differentiated from AGEP [1]. The primary clinical differential diagnosis of AGEP remains pustular psoriasis (von Zumbusch type), and often presents with a positive history of psoriasis, more generalised rash distribution, longer durations of pustules and fever, lack of a history of drug reactions or recent drug administration, more arthritis, and papillomatosis plus acanthosis on histology [1].

Sidoroff et al. [1] developed a sophisticated AGEP validation scoring system based on the EuroSCAR study group. We noted that our patient scored 10 out of a possible 12 on this scale, which they have denoted as 'definitely AGEP'. His cutaneous symptoms included an acute-onset rash on the face and flexures, and oedematous erythema, which then developed into a widespread rash consisting of hundreds of small $(<5 \mathrm{~mm})$ non-follicular sterile pustules. This was accompanied by the classic symptoms of fever $>38^{\circ} \mathrm{C}$, leucocytosis mostly due to neutrophilia $>7 \times 10^{9} /$ l and lymphadenopathy.

Whilst his recent sexual history remains a red flag, we acknowledge that $90 \%$ of cases are attributable to drugs [3], although the causative agent in this case is not confirmed. The patient's cold and flu medication in Bali remains unknown but likely contained a sympathomimetic agent such as pseudoephedrine and anti-pyretic/analgesic agent such as paracetamol, both of which have been known to cause AGEP [4]. However, we assume that this presentation could more likely be caused by the use of ibuprofen premorbidly, and his flareup after his discharge, when he consumed more ibuprofen, somewhat confirms this.

The patient's initial histopathology was not conclusive and did not fit with the clinical impression of the treating team, which may relate to either the disease process evolving, sampling error, or the lack of specific guidance to look for AGEP features by the pathologist. However, the second set of biopsies did show the typical features of AGEP including: spongiform subcorneal and/or intra-epidermal pustules, an often marked oedema of the papillary dermis, and perivascular infiltrates with neutrophils and exocytosis of some eosinophils [5]. Vasculitis and/or some single-cell necroses of keratinocytes may be present [6] (acute vasculitis demonstrated in foot biopsy). Psoriatic changes like acanthosis and papillomatosis are usually absent [6].

\section{Conclusion}

We presented this case to demonstrate that the combination of a diffuse pustular rash in combination with high fever and leucocytosis is often misinterpreted as an acute infectious 
Blake et al.: A Case of Acute Generalised Exanthematous Pustulosis after Unprotected Sexual Intercourse on Holidays in Bali

disease. It is important to diagnose AGEP early to avoid unnecessary and/or expensive investigations and treatments. It also demonstrates that AGEP is largely a clinical diagnosis and clinicians may wish to use the AGEP validation score [1] to assist in their diagnosis.

\section{References}

-1 Sidoroff A, Halevy S, Bavinck JN, Vaillant L, Roujeau JC: Acute generalized exanthematous pustulosis (AGEP): a clinical reaction pattern. J Cutan Pathol 2001;28:113.

$\checkmark 2$ Speeckaert MM, Speeckaert R, Lambert J, Brochez L: Acute generalized exanthematous pustulosis: an overview of the clinical, immunological and diagnostic concepts. Eur J Dermatol 2010;20:425-433.

-3 Roujeau JC, Bioulac-Sage P, Bourseau C, et al: Acute generalized exanthematous pustulosis. Analysis of 63 cases. Arch Dermatol 1991;127:1333-1338.

4 Saissi EH, Beau-Salinas F, Jonville-Béra AP, Lorette G, Autret-Leca E: Drugs associated with acute generalized exanthematic pustulosis. Ann Dermatol Venereol 2003;130:612-618.

5 Burrows NP, Russell Jones RR: Pustular drug eruptions: a histopathological spectrum. Histopathology 1993;22:569.

6 Beylot C, Doutre MS, Beylot-Barry M: Acute generalized exanthematous pustulosis. Semin Cutan Med Surg $1996 ; 15: 244$.

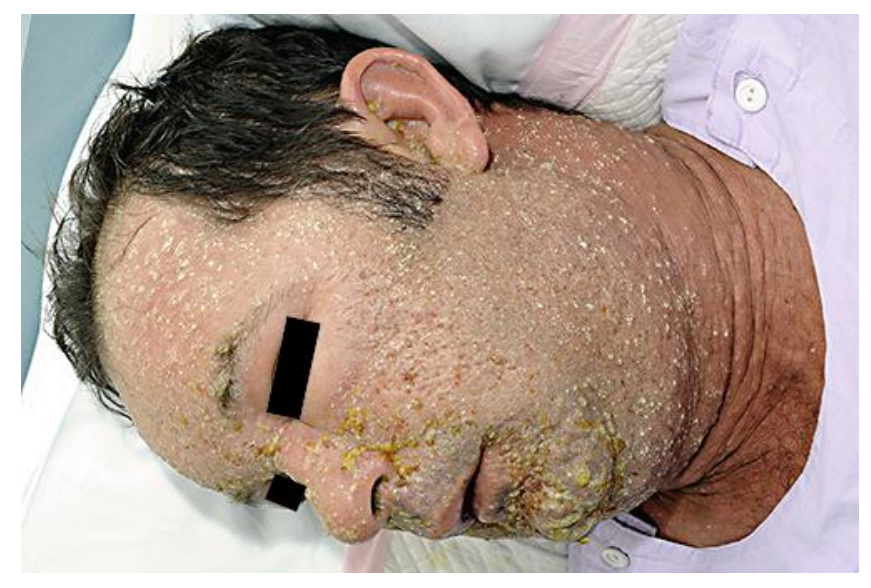

Fig. 1. Facial erythema, oedema and numerous pustules with crusting around the nose and mouth. 
Blake et al.: A Case of Acute Generalised Exanthematous Pustulosis after Unprotected Sexual Intercourse on Holidays in Bali

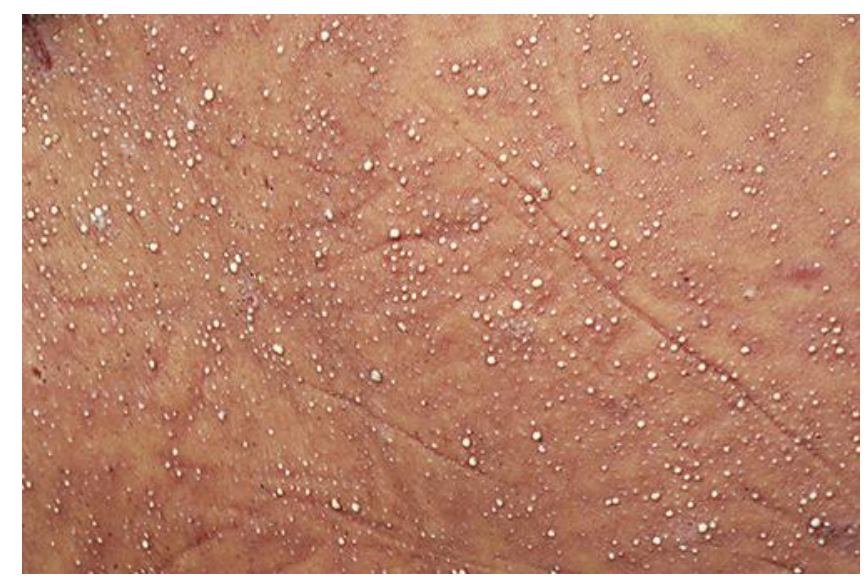

Fig. 2. Close-up of the back: non-follicular pustules on an erythematous base.

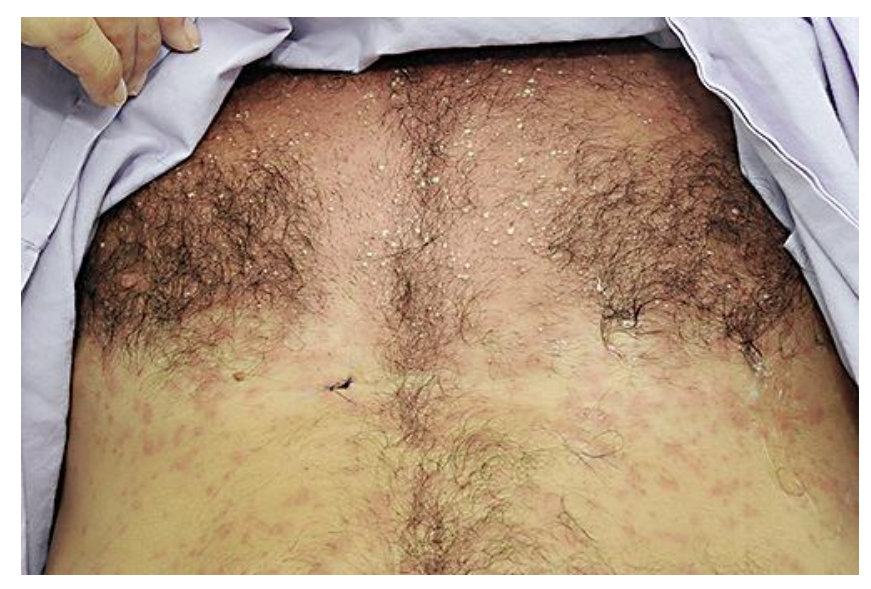

Fig. 3. Chest: pustules on an erythematous base. 